La

Révolution

française

\section{La Révolution française}

Cahiers de l'Institut d'histoire de la Révolution française

13 | 2018

Pratiques et enjeux scientifiques, intellectuels et politiques de la traduction (vers 1660-vers 1840)

\title{
Les traductions des textes techniques destinées aux officiers des armes savantes (Italie-France / France-Italie, 1750-1840)
}

Lorenzo Cuccoli

\section{OpenEdition}

\section{Journals}

Édition électronique

URL : http://journals.openedition.org/lrf/1940

DOI : $10.4000 /$ Irf. 1940

ISSN : 2105-2557

\section{Éditeur}

IHMC - Institut d'histoire moderne et contemporaine (UMR 8066)

Référence électronique

Lorenzo Cuccoli, «Les traductions des textes techniques destinées aux officiers des armes savantes (Italie-France / France-Italie, 1750-1840) », La Révolution française [En ligne], 13 | 2018, mis en ligne le 22 janvier 2018, consulté le 19 avril 2019. URL : http://journals.openedition.org//rf/1940 ; DOI : 10.4000/Irf.1940

Ce document a été généré automatiquement le 19 avril 2019

(C) La Révolution française 


\title{
Les traductions des textes techniques destinées aux officiers des armes savantes (Italie-France / France-Italie, 1750-1840)
}

\author{
Lorenzo Cuccoli
}

Je tiens à remercier Patrice Bret et Maria Pia Casalena pour leurs précieuses suggestions.

1 La littérature technique militaire a joué un rôle important pour accompagner les réformes des armes savantes, de leur enseignement et de leurs matériels dans la seconde moitié $d u \mathrm{XVIII}^{\mathrm{e}}$ siècle et les premières décennies $d u \mathrm{XIX}^{\mathrm{e}}$ siècle, époque à laquelle la France a constitué un modèle avec l'École du génie de Mézières, fondée en 1748, et l'artillerie nouvelle de Gribeauval, développée et mise en place, non sans une forte résistance, au lendemain de la Guerre de Sept Ans ${ }^{1}$. Il en est de même d'ouvrages hostiles aux doctrines en place, qui n'ont pas trouvé en France un écho suffisant pour les supplanter, mais ont pu rencontrer un succès plus grand à l'étranger, comme la «fortification perpendiculaire» de Montalembert en Allemagne ou aux États-Unis ${ }^{2}$. La traduction a joué un rôle non négligeable dans la diffusion de ces ouvrages, mais l'historiographie a porté peu d'attention aux traductions de textes techniques militaires en général, pourtant fort nombreuses, à leur genèse et à leur impact, comme à la personnalité des traducteurs et à leurs motivations ${ }^{3}$.

2 Ce silence est particulièrement regrettable pour les traductions réalisées dans ce domaine, dans les deux sens, entre la France et l'Italie, pour éclairer les échanges et les influences mutuelles entre ces espaces linguistiques, à cette époque de bouleversements politiques et militaires qui allaient conduire aux guerres révolutionnaires et impériales et, à terme, à l'unité italienne. En revanche, depuis peu, l'historiographie italienne a commencé à s'intéresser concrètement aux médiations et aux transferts culturels transnationaux entre la seconde moitié du $\mathrm{XVIII}^{\mathrm{e}}$ siècle et le Risorgimento ${ }^{4}$. En nous 
insérant dans cette tradition d'études, nous proposons ici une étude de cas des circulations et transferts entre la France et la péninsule italienne, plus spécialement consacrée aux dynamiques relatives aux corps savants entre le milieu du XVIII ${ }^{\mathrm{e}}$ siècle et celui du XIX ${ }^{\mathrm{e}}$. Comment ces traductions participent-elles des dynamiques des Lumières, de la Révolution française et de l'émergence du Risorgimento?

3 Après avoir présenté les logiques d'établissement du corpus en cherchant à identifier d'éventuelles spécificités des traductions à l'usage des armes savantes, nous examinerons tour à tour celles du XVIII ${ }^{\mathrm{e}}$ siècle, antérieures à la Révolution française, puis celles du XIX siècle. Enfin, par l'étude de traductions des années 1830 faites par des civils, nous chercherons à vérifier la validité de spécificités de la traduction militaire à cette époque.

\section{Établissement et analyse du corpus}

Cette étude porte sur les traductions de l'italien en français et du français en italien des textes techniques destinés aux officiers de l'artillerie et du génie entre 1750 et 1840 . Les traductions de l'italien en français ont été repérées dans les recueils publiés par Giovanni Dotoli et Vito Castiglione Minischetti pour les $\mathrm{XVIII}^{\mathrm{e}}$ et $\mathrm{XIX}^{\mathrm{e}}$ siècles ${ }^{5}$. La liste des traductions du français en italien a été établie à partir du catalogue unique italien ICCU6.

Les principes suivants ont présidé à l'inclusion d'un ouvrage dans le corpus. Pour les textes eux-mêmes : tout d'abord, seules les monographies ont été retenues, à l'exclusion des articles de périodiques; ensuite, l'ouvrage devait être "technique» dans une acception large, en incluant les manuels de service, de physique appliquée, de balistique et d'ingénierie, mais en excluant les mathématiques pures ${ }^{7}$; enfin, il devait être destiné, dans sa traduction et, de préférence, pour l'original, aux officiers ou élèves du génie ou de l'artillerie. Quant aux traducteurs, ils devaient être officiers de ces armes savantes ou à défaut officiers d'une autre arme ayant un intérêt pour la technique : Pampani et Zener constituent des "hybrides», le premier appartenant aux ingénieurs-géographes, qui étaient liés au génie, et le deuxième étant capitaine des milices, qui contenaient des bombardiers, tandis que de Flavigny appartenait à la cavalerie. Les auteurs eux-mêmes se révèlent être des officiers des armes savantes ou des professeurs des écoles militaires. On est donc en présence d'œuvres qui, avec des exceptions, circulaient dans une sorte de circuit clos au sein des corps respectifs.

6 À ce corpus intègre sont joints également deux ouvrages de Bernard Forest de Bélidor (1693-1761) qui ont été traduits en italien par des civils. Cette apparente exception aux critères généraux de sélection pour des textes de la première moitié du XVIII siècle, qui connaissent une nouvelle actualité avec des rééditions commentées au début du XIX siècle, s'explique par leur importance et par l'intérêt que représente l'utilisation, par des ingénieurs civils, de textes destinés aux ingénieurs militaires. Ce cas particulier nous permet également, in fine, de vérifier la spécificité, ou non, de la traduction militaire dans les textes techniques destinés aux officiers des armes savantes.

Tableau 1 - Traductions de l'italien en français

\begin{tabular}{|l|l|l|l|}
\hline Auteur & Traducteur & Traduction & Édition originale \\
\hline
\end{tabular}




\begin{tabular}{|c|c|c|c|}
\hline $\begin{array}{l}\text { Papacino } \\
\text { d'Antoni }\end{array}$ & Flavigny & $\begin{array}{l}\text { Examen de la poudre, Amsterdam, } \\
\text { Marc-Michel Rey, } 1773\end{array}$ & $\begin{array}{l}\text { Esame della polvere, Torino, } \\
\text { Stamperia reale, } 1765\end{array}$ \\
\hline $\begin{array}{l}\text { Papacino } \\
\text { d'Antoni }\end{array}$ & Flavigny & $\begin{array}{l}\text { Principes fondamentaux de la } \\
\text { construction des places, avec des } \\
\text { Réflexions propres à démontrer les } \\
\text { perfections \& les imperfections de celles } \\
\text { qui sont construites; un nouveau } \\
\text { Système de Fortification sur toute } \\
\text { espèce de ligne; \& une nouvelle Théorie } \\
\text { des Mines, Londres et Paris, Ruault - } \\
\text { Jombert le fils - L'Esprit, } 1775\end{array}$ & $\begin{array}{l}\text { Dell'architettura militare per le } \\
\text { regie scuole teoriche d'artiglieria, } \\
\text { e fortificazione. Libro terzo in cui } \\
\text { si contengono le regole della } \\
\text { fortificazione difensiva, e delle } \\
\text { mine per le piazze di guerra, } \\
\text { Torino, Stamperia reale, } 1759\end{array}$ \\
\hline $\begin{array}{l}\text { Papacino } \\
\text { d'Antoni }\end{array}$ & $\begin{array}{l}\text { [Cusset de } \\
\text { Montrozard] }\end{array}$ & $\begin{array}{l}\text { Institutions physico-méchaniques, à l' } \\
\text { usage des écoles royales d'artillerie et du } \\
\text { génie de Turin, Strasbourg, Bauer et } \\
\text { Treuttel, } 1777,2 \text { vol. }\end{array}$ & $\begin{array}{l}\text { Instituzioni fisico-meccaniche per } \\
\text { le regie scuole d'artiglieria e } \\
\text { fortificazione, } \\
\text { Stamperia reale, } 1773-1774,2 \\
\text { vol. }\end{array}$ \\
\hline $\begin{array}{l}\text { Papacino } \\
\text { d'Antoni }\end{array}$ & $\begin{array}{l}\text { Cusset de } \\
\text { Montrozard }\end{array}$ & $\begin{array}{l}\text { Du Service de l'artillerie à la guerre, } \\
\text { Paris, L. Cellot, } 1780\end{array}$ & $\begin{array}{l}\text { Dell'artiglieria pratica per le } \\
\text { Regie Scuole d'artiglieria, t. II, } \\
\text { Incumbenze degli artiglieri in } \\
\text { tempo di guerra, Torino, } \\
\text { Stamperia Reale, } 1775\end{array}$ \\
\hline $\begin{array}{l}\text { Papacino } \\
\text { d'Antoni }\end{array}$ & Saint-Auban & $\begin{array}{l}\text { De l'Usage des armes à feu, Paris, } \\
\text { Lambert, } 1785\end{array}$ & $\begin{array}{l}\text { Dell'uso delle armi da fuoco per le } \\
\text { regie scuole teoriche d'artiglieria, } \\
\text { e fortificazione, Torino, } \\
\text { Stamperia Reale, } 1780\end{array}$ \\
\hline
\end{tabular}

Tableau 2 - Traductions du français en italien

\begin{tabular}{|c|c|c|c|}
\hline Auteur & Traducteur & Traduction & Édition originale \\
\hline Le Blond & Zener & $\begin{array}{l}\text { L'artiglieria per principj, e per } \\
\text { raziocinio. Trattato che contien la } \\
\text { descrizione, e l'uso delle diverse } \\
\text { bocche da fuoco, e i principali } \\
\text { mezzi, che si sono adoperati per } \\
\text { perfezionarle, La Teoria, e la } \\
\text { pratica delle Mine, e del Getto delle } \\
\text { Bombe, e tutto ciò, che l'Artiglieria } \\
\text { ha di più importante dopo il trovato } \\
\text { della polvere da Cannone, Venezia, } \\
\text { Antonio Locatelli, 1773, } 2 \text { vol. }\end{array}$ & $\begin{array}{l}\text { L'Artillerie raisonnée, contenant la } \\
\text { description \& l'usage des différentes } \\
\text { bouches à feu, avec les principaux } \\
\text { moyens qu'on a employés pour les } \\
\text { perfectionner, la théorie \& la } \\
\text { pratique des mines, \& du jet des } \\
\text { bombes, et l'essentiel de tout ce que } \\
\text { l'artillerie a de plus intéressant } \\
\text { depuis l'invention de la poudre a } \\
\text { canon, Paris, C.-A. Jombert, } 1771\end{array}$ \\
\hline
\end{tabular}




\begin{tabular}{|c|c|c|c|}
\hline Anonyme & Anonyme & $\begin{array}{l}\text { Piccolo manuale del cannoniere } \\
\text { ossia istruzione generale } \\
\text { concernente il servigio di tutte le } \\
\text { bocche da fuoco in uso } \\
\text { nell'artiglieria tradotto dal francese } \\
\text {, Milano, Margaillan, } 1804\end{array}$ & $\begin{array}{l}\text { Petit manuel du canonnier, ou } \\
\text { instruction générale sur le service } \\
\text { de toutes les bouches à feu, en } \\
\text { usage dans l'artillerie, Paris, } \\
\text { Magimel, An II (1793-1794) }\end{array}$ \\
\hline Dembarrère & Pampani & $\begin{array}{l}\text { Colpo d'occhio Per distinguere, e } \\
\text { classificare le differenti parti della } \\
\text { Scienza Militare, o dell'Arte della } \\
\text { Guerra sopra terra, ed in } \\
\text { particolare per riconoscere l' } \\
\text { estensione e l'influenza di quelle, } \\
\text { che appartengono all'arma del } \\
\text { Genio, Pavia, Bolzani, } 1805\end{array}$ & $\begin{array}{l}\text { Coup d'œil pour distinguer et } \\
\text { classer les différentes parties de la } \\
\text { science militaire ou de l'Art de la } \\
\text { guerre sur terre, surtout pour } \\
\text { reconnoître l'étendue et l'influence } \\
\text { de celles qui appartiennent à l'arme } \\
\text { du génie, Paris, Magimel, } 1801\end{array}$ \\
\hline Le Blond & Corso & $\begin{array}{l}\text { Memorie d'artiglieria, Pavia, } \\
\text { Giovanni Capelli, } 1805\end{array}$ & $\begin{array}{l}\text { Traité de l'Artillerie, ou des Armes } \\
\text { et Machines en usage à la Guerre, } \\
\text { Depuis l'invention de la Poudre, } \\
\text { Paris, C.-A. Jombert, } 1743\end{array}$ \\
\hline Le Blond & Corso & Ibidem & $\begin{array}{l}\text { L'Artillerie raisonnée, contenant la } \\
\text { description \& l'usage des différentes } \\
\text { bouches à feu, avec les principaux } \\
\text { moyens qu'on a employés pour les } \\
\text { perfectionner, la théorie \& la } \\
\text { pratique des mines, \& du jet des } \\
\text { bombes, et l'essentiel de tout ce que } \\
\text { l'artillerie a de plus intéressant } \\
\text { depuis l'invention de la poudre à } \\
\text { canon, Paris, C.-A. Jombert, } 1771\end{array}$ \\
\hline Le Blond & Corso & $\begin{array}{l}\text { Ibidem [diverses autres } \\
\text { traductions }{ }^{8} \text { ] }\end{array}$ & [divers autres mémoires] \\
\hline Lombard & Pacces & $\begin{array}{l}\text { Trattato del moto de' projetti } \\
\text { applicato al tiro delle bocche a } \\
\text { fuoco, Napoli, Masi, } 1816\end{array}$ & $\begin{array}{l}\text { Traité du mouvement des } \\
\text { projectiles, appliqué au tir de } \\
\text { bouches à feu, Dijon, Imprimerie } \\
\text { de L. N. Frantin, } 1796\end{array}$ \\
\hline Lombard & Pacces & $\begin{array}{l}\text { Tavole del tiro de' cannoni e degli } \\
\text { obici con una istruzione sulla } \\
\text { maniera di servirsene, Napoli, } \\
\text { Masi, } 1816\end{array}$ & $\begin{array}{l}\text { Tables du tir des canons et des } \\
\text { obusiers, avec une instruction sur } \\
\text { la manière de s'en servir, s.l., } 1787\end{array}$ \\
\hline $\begin{array}{l}\text { Poumet (ou } \\
\text { Augoyat) }\end{array}$ & $\begin{array}{l}\text { Biondi } \\
\text { Perelli }\end{array}$ & $\begin{array}{l}\text { Istruzione sulla balistica, Livorno, } \\
\text { Pozzolini, } 1827\end{array}$ & $\begin{array}{l}\text { Instruction sur la balistique, à l' } \\
\text { usage des élèves du Corps royal d' } \\
\text { État-major, Paris, Anselin et } \\
\text { Pochard, } 1824\end{array}$ \\
\hline
\end{tabular}




\begin{tabular}{|c|c|c|c|}
\hline $\begin{array}{l}\text { von Decker } \\
\text { [trad. } \\
\text { Ravichio de } \\
\text { Peretsdorf et } \\
\text { Nancy] }\end{array}$ & $\begin{array}{l}\text { Biondi } \\
\text { Perelli }\end{array}$ & $\begin{array}{l}\text { Trattato elementare d'artiglieria } \\
\text { per l'uso dei militari di tutte le armi } \\
\text {, Livorno, Giulio Sardi, 1830, } 3 \\
\text { vol. }\end{array}$ & $\begin{array}{l}\text { Traité élémentaire d'artillerie, à l' } \\
\text { usage des militaires de toutes les } \\
\text { armes, Paris, F.-G. Levrault, 1825, } \\
3 \text { vol. }\end{array}$ \\
\hline Lamy & Novi & $\begin{array}{l}\text { Trattato teorico e pratico delle } \\
\text { batterie, Napoli, Reale Tipografia } \\
\text { della Guerra, } 1830\end{array}$ & $\begin{array}{l}\text { Traité théorique et pratique } \\
\text { des Batteries, Paris, Anselin et } \\
\text { Pochard, } 1827\end{array}$ \\
\hline Savart & $\begin{array}{l}\text { Biondi } \\
\text { Perelli }\end{array}$ & $\begin{array}{l}\text { Corso elementare di fortificazione } \\
\text { ad uso delle scuole militari, } \\
\text { Livorno, Giulio Sardi, 1830-1831, } \\
4 \text { vol. }\end{array}$ & $\begin{array}{l}\text { Cours élémentaire de fortification, à } \\
\text { l'usage de MM. les élèves de l'École } \\
\text { spéciale militaire, Paris, Anselin } \\
\text { et Pochard, 1825, } 4 \text { vol. }\end{array}$ \\
\hline $\begin{array}{l}\text { Anonyme } \\
\text { [trad. } \\
\text { Ravichio de } \\
\text { Peretsdorf] }\end{array}$ & $\begin{array}{l}\text { Biondi } \\
\text { Perelli }\end{array}$ & $\begin{array}{l}\text { Trattato di pirotecnia militare } \\
\text { comprendente tutti i fuochi } \\
\text { artifiziati da guerra. Versione } \\
\text { italiana con riduzione di pesi e } \\
\text { misure, Livorno, Giulio Sardi, } \\
1831\end{array}$ & $\begin{array}{l}\text { Traité de pyrotechnie militaire, } \\
\text { comprenant tous les artifices de } \\
\text { guerre en usage en Autriche, Paris, } \\
\text { F.-G. Levrault, } 1824\end{array}$ \\
\hline
\end{tabular}

Tableau 3 - Traductions du français en italien par des civils

\begin{tabular}{|l|l|l|l|}
\hline Auteur & Traducteur & Traduction & Édition originale \\
\hline Bélidor & Masieri & $\begin{array}{l}\text { La scienza degli ingegneri nella direzione } \\
\text { delle opere di fortificazione e d'architettura } \\
\text { civile, Mantova, Fratelli Negretti - Milano, } \\
\text { Gaspare Truffi, 1832, 2 vol. - Firenze, } \\
\text { presso gli editori, 1832, puis Livorno, } \\
\text { Giulio Sardi, 1834 }\end{array}$ & $\begin{array}{l}\text { La Science des ingénieurs dans la } \\
\text { fonduite des travaux de } \\
\text { civile, Paris, F. Didot frères, } \\
1830\end{array}$ \\
\hline Bélidor & Soresina & $\begin{array}{l}\text { Architettura idraulica: ovvero Arte di di } \\
\text { condurre, innalzare e regolare le acque pei } \\
\text { vari bisogni della vita, Mantova, fratelli } \\
\text { Negretti - Firenze, presso gli editori, } \\
\text { 1835-1839, 4 vol. }\end{array}$ & $\begin{array}{l}\text { Architecture hydraulique, ou L' } \\
\text { art de conduire, d'élever et de } \\
\text { ménager les eaux pour les } \\
\text { différens besoins de la vie, Paris, } \\
\text { F. Didot, 1819, 2 vol. }\end{array}$ \\
\hline
\end{tabular}

\section{Analyse du corpus}

7 Le corpus retenu - seize ouvrages traduits dans les deux sens, auxquels il faut rajouter les deux de Bélidor, que l'on considérera à part dans le corpus - ne prétend pas former un recensement exhaustif, mais il est pleinement significatif en ce qu'il fournit une idée du nombre congrue des traductions dans ce domaine ${ }^{9}$. Il est remarquable que cinq seulement de ces ouvrages soient traduits de l'italien vers le français, que leur publication soit restreinte à une courte période d'une douzaine d'années (entre 1773 à 1785), et qu'ils 
concernent un même et unique auteur (tableau 1). Au contraire, les onze traductions du français en italien (tableau 2) s'étalent sur l'ensemble de la période en se regroupant souvent autour de moments et d'espaces particuliers: une seule traduction dans la seconde moitié du XVIII ${ }^{\mathrm{e}}$ siècle à Venise; trois entre 1804 et 1805 dans la République italienne et le Royaume d'Italie, dont un recueil de plusieurs mémoires publiés séparément dans la langue d'origine ; deux en 1816 dans le Royaume des Deux-Siciles; et six entre 1827 et 1831, surtout en Toscane, auxquelles il faut ajouter les deux traductions de Bélidor, de 1832 à 1839 dans le Royaume Lombardo-Vénitien et en Toscane. Tous les principaux anciens états italiens étaient donc concernés, sauf le Piémont et les États du Pape. Comparé à l'ensemble des traductions en langue française, ce corpus peut sembler assez restreint, mais il est plutôt consistant par rapport au total des ouvrages traduits en langue italienne. Le cadre temporel parait approprié pour l'identification de la France comme modèle dans le domaine des armes savantes - modèle qui deviendra politiquement suspect à partir de la Révolution française. Il en est de même pour la centralité de la figure de l'ingénieur (y compris l'artilleur) - centralité certes reconnue en France, notamment depuis la Révolution, beaucoup moins dans la plupart des états italiens, cependant avec une intégration progressive dans la vie publique et scientifique justement à partir des années 1830 .

marque une majorité d'ouvrages plutôt dédiés aux artilleurs, avec douze ouvrages traitant de balistique ${ }^{10}$ et du service du canon ${ }^{11}$; un manuel de physique et mécanique est explicitement destiné aux élèves des deux corps ${ }^{12}$; tandis que seulement trois œuvres relatives surtout à l'architecture militaire et à la fortification sont réservées au génie ${ }^{13}$, auxquelles on peut rajouter les deux traités de Bélidor.

Concernant les auteurs traduits, le célèbre artilleur et savant piémontais Alessandro Vittorio Papacino d'Antoni (1714-1786), directeur des écoles royales d'artillerie et de fortifications de Turin, est non seulement le seul auteur italien à être traduit, mais également celui qui est le plus traduit, avec cinq ouvrages différents. En second lieu, Guillaume Le Blond (1704-1781), professeur de mathématiques des Enfants de France, est traduit deux fois, en 1773 et 1805, ainsi que l'ingénieur militaire Bernard Forest de Bélidor, de 1832 à 1839 . Tous les autres auteurs n'ont été traduits qu'une fois. Il s'agit majoritairement d'officiers des corps savants : pour le génie, le général de division Jean Dembarrère (1747-1828) ; pour l'artillerie, l'ancien colonel Joseph Ravichio de Peretsdorf $(1767-1844)^{14}$, les capitaines Benjamin Poumet ${ }^{15}$ et Jean-Nicolas Lamy. On retrouve aussi un capitaine d'infanterie, mais professeur de fortification à l'École spéciale militaire, Nicolas-Pierre-Antoine Savart (1765-c.1825). Karl von Decker (1784-1844), enfin, était quant à lui major dans l'état-major prussien.

Les traductions concernent autant des ouvrages relativement récents que des ouvrages plus anciens, avec une moyenne un peu supérieure à dix ans et un minimum de deux ans ${ }^{16}$

Quant aux traducteurs, le plus prolifique est le lieutenant d'artillerie Ferdinando Biondi Perelli, avec quatre ouvrages. Suivent trois lieutenants-colonels, avec deux traductions chacun : pour la cavalerie, Gratien-Jean-Baptiste-Louis Vicomte de Flavigny ${ }^{17}$ (1740-1783), et, pour l'artillerie, le chevalier Cusset de Montrozard $^{18}$ et Giovanni Battista Pacces, également auteur d'un traité sur les batteries ${ }^{19}$. Le cas de loin le plus fréquent est celui des militaires qui ne traduisirent qu'un ouvrage: on a cité les cas «hybrides» des capitaines Gabriele Zener et Giulio Pampani, auquel on doit aussi deux écrits d'arpentage et d'ingénierie hydraulique ${ }^{20}$; on retrouve ensuite des officiers d'artillerie : le lieutenant- 
général Jacques-Antoine Baratier, marquis de Saint-Auban, et les capitaines Antonio Corso et Pietro Novi. L'unique traducteur anonyme, celui du Petit manuel du canonnier, était très probablement un officier d'artillerie, compte tenu du caractère strictement technique de ce texte - d'où la décision de l'intégrer dans le corpus. Les traducteurs civils de Bélidor - Luigi Masieri et Basilio Soresina - étaient docteurs en physique et mathématiques.

12 Si l'on examine les grades des traducteurs, on remarque une bipartition nationale étonnante : si les trois traducteurs français sont tous des officiers supérieurs, cinq des six traducteurs italiens sont des officiers subalternes, lieutenants ou capitaines - seul Pacces fait exception. Cela nous renseigne sur les motivations probables des traducteurs : pour les officiers de grade inférieur, une traduction utile pouvait sans doute faire espérer une promotion. Ainsi trouve-t-on fréquemment, pour les traducteurs italiens, des dédicaces à un personnage puissant, directement lié aux promotions, tel le commandant du corps ou le ministre de la guerre ${ }^{21}$. Indépendamment de leur grade, les traducteurs pouvaient entreprendre des traductions à l'usage des écoles militaires, soit parce qu'ils étaient euxmêmes responsables de l'instruction (c'est le cas de Pacces ${ }^{22}$ et de Biondi Perelli), soit sous l'impulsion de leurs supérieurs (c'est le cas de Montrozard ${ }^{23}$ ), les deux motivations pouvant évidemment coexister chez le même traducteur.

13 La distribution des traductions appelle plusieurs remarques. Il faut souligner au préalable l'énorme écart quantitatif d'imprimés à caractère militaire entre la France et l'Italie. La culture militaire française, et particulièrement celle des armes savantes, était dominante en Europe et, donc, dans la péninsule pendant la période considérée, même eu égard aux différentes politiques conduites en la matière par les États italiens. La production «italienne» dans ce domaine était au contraire assez limitée, d'où l'absence de traductions de l'italien en français, à l'exception des cinq ouvrages de l'artilleur et savant piémontais d'Antoni, qui eut une renommée européenne. Le faible développement des publications militaires en Italie, en raison du faible intérêt des États italiens pour les questions militaires avant les guerres de la Révolution - si l'on excepte justement le Piémont - peut expliquer l'absence quasi-totale de traductions du français vers l'italien dans ce domaine, ainsi que la connaissance du français, longtemps langue hégémonique en Europe, parmi les officiers des armes savantes. La « rééducation militaire » de l'Italie passa donc par l'influence française, ce dont témoignent les quelques traductions d'ouvrages, sorties entre 1804 et 1805, et même les deux traductions napolitaines de 1816, qui parurent peu après la mort du roi Joachim-Napoléon Murat. Le dernier groupe de traductions (six entre 1827 et 1831) parait surtout lié à l'initiative de l'artillerie toscane. Cependant, on peut y voir également le reflet du renouvellement de la science italienne dans ces années-là, dont la Toscane était alors le centre d'impulsion ${ }^{24}$. L'adaptation des œuvres de Bélidor pour l'ingénierie civile dans la même Toscane et en Lombardie dans les années 1830 s'inscrit dans la même dynamique et paraît être liée au processus de professionnalisation des ingénieurs ${ }^{25}$.

14 Notons également que le corpus retenu révèle à la fois la croissance et les tendances du marché du livre : si, en France, la plupart des traductions sont éditées à Paris, en Italie, la seule traduction du $\mathrm{XVIII}^{\mathrm{e}}$ siècle sort à Venise, principal centre pour l'imprimerie de langue italienne ; Milan prend la relève à partir de l'époque napoléonienne et jusqu'aux années 1840 , ce qui se reflète dans notre corpus avec trois ouvrages sortis entre Milan et la ville universitaire de Pavie, située à proximité, pendant la période napoléonienne ; trois textes témoignent de la vitalité de Naples et quatre de celle de Livourne, en Toscane, un 
centre important à la fois pour les échanges culturels et pour la circulation des imprimés ${ }^{26}$. La Lombardie et la Toscane sont au premier plan également pour les traductions de Bélidor.

\section{Les spécificités de l'édition militaire}

15 Afin de bien comprendre les enjeux de la traduction de ce type de texte, il faut d'abord se placer dans le contexte spécifique de l'édition militaire. La décision de publier un texte à caractère militaire ne dépendait pas seulement de sa qualité et de son intérêt auprès du public, mais aussi de son opportunité par rapport à un contexte - qu'il soit national, international, éditorial. Il s'agissait, parfois, de la circulation de savoirs d'État. Ainsi, la situation de guerre ou de paix (au moins continentale) joue un rôle essentiel, comme la position de l'auteur par rapport aux institutions militaires: Est-il intégré dans ces dernières? Vise-t-il une intégration? Se positionne-t-il en contestateur? En tout cas, les imprimés ne sont que la face visible de centaines de manuscrits qui circulaient au sein des corps; de même, les traductions d'imprimés étrangers ne sont qu'une partie de celles qui pouvaient être commissionnées mais qui restaient souvent manuscrites. Pour les officiers des armes savantes, plus scolarisés et à qui le service demandait le plus de connaissances scientifiques et techniques, la circulation de textes était particulièrement intense et absolument nécessaire. D'ailleurs, cette même scolarisation jouait en faveur de la connaissance des langues française et italienne et de la lecture de l'original quand il pouvait être disponible. Aussi n'est-il pas surprenant de trouver un faible nombre de traductions, par rapport à la quantité globale d'imprimés en la matière. En revanche, la traduction était aussi le moyen de rendre les ouvrages plus aisément disponibles dans un pays étranger.

16 L'intérêt des traductions rédigées par ces officiers réside dans le fait qu'ils faisaient quotidiennement face à des opérations de «traduction » : en tant qu'intermédiaires dans la hiérarchie militaire et technique, ils devaient constamment traduire des réflexions, des ordres, des instructions, des rapports ou des projets, etc., et interpréter la réalité en utilisant leurs langues propres, celles des sciences et du dessin. Typique de ces corps savants, l'interpénétration permanente entre pratiques et fonctions militaires, d'un côté, et pratiques et fonctions technico-scientifiques, de l'autre, portait également ces officiers à un curieux mélange entre le respect de l'autorité, qu'elle soit militaire ou scientifique, et la nécessité de la critique. Largement dominant dans les traductions que nous avons examinées, le respect de l'autorité est marqué très souvent par l'effacement du traducteur et sa fidélité scrupuleuse au texte original. Les observations et les critiques sont très rares et réservées au paratexte. En l'occurrence, cela concorde parfaitement avec les conclusions des historiens qui ont travaillé sur les traductions en général ou sur la censure au cours de la même période ${ }^{27}$. Il n'y aurait donc pas, à ce titre, de spécificité de la traduction technique militaire.

\section{La domination des traductions de l'italien au XVIII siècle}

17 On traduit donc davantage du français vers l'italien qu'en sens inverse et, cependant, on observe une bipartition parmi le $\mathrm{XVIII}^{\mathrm{e}}$ et le $\mathrm{XIX}^{\mathrm{e}}$ siècle : au $\mathrm{XVIII}^{\mathrm{e}}$, le sens prévalent est 
celui de l'italien en français, tandis que, au XIX ${ }^{\mathrm{e}}$, cette veine se tarit, avec une inversion de tendance marquée qui, de toute évidence, est liée à la présence française en Italie.

\section{Plusieurs traducteurs français pour un unique auteur italien : Papacino d'Antoni}

Le premier agrégat est constitué par les traductions de l'italien en français, sorties entre 1773 et 1785 dans le contexte de la «révolution balistique » et des débats autour des réformes de Gribeauval. Il s'agit de cinq ouvrages d'un même auteur : directeur des écoles royales d'artillerie et de fortifications de Turin, Papacino d'Antoni est devenu célèbre pour ses expériences sur la balistique intérieure et extérieure, qui s'inscrivent dans cette « révolution balistique » du XVIII ${ }^{\mathrm{e}}$ siècle, fondée sur les travaux de Benjamin Robins et Leonhard Euler et dont les écoles de Turin furent les premières en Europe à accueillir le « changement de paradigme ${ }^{28}$ ». En effet, la balistique en était transformée d'une science galiléenne qui n'était applicable qu'aux mortiers à une science newtonienne applicable à toutes les bouches à feu. Cela impliquait une emphase nouvelle posée dans la préparation des artilleurs sur le calcul différentiel et intégral, particulièrement visible dans le texte le plus théorique, les Institutions physico-méchaniques.

L'importance des expériences de Papacino d'Antoni est remarquée par le marquis de Saint-Auban (1712-1783), un proche de Buffon, Franklin, Condorcet et Perronet, vétéran des guerres de Succession de Pologne et d'Autriche et de la guerre de Sept Ans ${ }^{29}$ :

On sera sans doute étonné du nombre et des différentes expériences qui ont été faites en grand sur chacun des objets, de la rigueur, de la précision et de l'exactitude qu'on y a employées, afin de pouvoir asseoir sur les résultats, des jugements positifs, irrévocables et sans retour. On ne sera pas moins étonné des sommes immenses qu'il en a dû en coûter pour faire en grand ces diverses épreuves. C'est ainsi cependant que les vérités se découvrent, que les Arts et les Sciences parviennent à un plus haut degré de perfection ${ }^{30}$.

Le vicomte de Flavigny souligne également la valeur scientifique de ces traductions au regard de l'autorité acquise dans le domaine militaire par leur auteur : « Les CEuvres de M. d'Antoni méritent d'être connues dans toutes les Langues et de tous les Savants ${ }^{31}$.» De fait, l'Examen de la poudre est présent dans la bibliothèque de savants tels que Lavoisier ${ }^{32}$, mais surtout, à l'exception de son traité de fortification, toutes ses traductions furent utilisées dans les écoles régimentaires françaises d'artillerie, et cela au moins jusque dans les premières décennies du XIX ${ }^{\mathrm{e}}$ siècle, puisque le Comité central de l'artillerie considère encore en 1820 qu'elles doivent figurer dans les bibliothèques de ces écoles ${ }^{33}$. Ces œuvres étaient donc considérées comme nécessaires pour la formation même des officiers, ce qui explique la nécessité de la traduction. Ceci est explicité notamment dans le «Discours Préliminaire» des Institutions physico-méchaniques, par Montrozard en 1777: "Ces Institutions Physico-Mécaniques correspondent parfaitement au plan d'instruction, qu'on a voulu établir dans nos écoles après la paix ${ }^{34}$. Il précise d'ailleurs que l'initiative de la traduction revenait à ses supérieurs, responsables de l'instruction des écoles ${ }^{35}$. En effet, cet ouvrage a incontestablement un but pédagogique, en privilégiant les expériences sur le calcul, malgré le recours, parfois, à des mathématiques de haut niveau, comme le calcul intégral ${ }^{36}$. La principale intervention personnelle du traducteur se limite au «Discours Préliminaire ", dans lequel il exprime son souhait d'imiter le modèle piémontais, alliant l'expérimentation à la formation mathématique poussée. Le texte lui-même est traduit fidèlement dans un style élégant, mais Montrozard y corrige néanmoins quelques 
formules mathématiques, tout en commettant lui-même des erreurs dans les conversions de mesure ${ }^{37}$... La traduction Du Service de l'artillerie à la guerre, du même Montrozard en 1780 , est la seule où l'on relève une mise à jour systématique du texte dans les notes de bas de page, car elle intègre la réforme du matériel français réalisée par Gribeauval. Le traducteur considère important de traduire cet ouvrage après tant d'années de paix, mais il s'engage en revendiquant explicitement la supériorité de l'«artillerie nouvelle» française par rapport aux modèles précédents ${ }^{38}$, notamment en termes de mobilité, en campagne, grâce au train d'artillerie («Rien n'est si roulant et en même temps si solide. Aussi l'artillerie peut aujourd'hui passer partout, et aller si vite que l'armée $\left.{ }^{39} . »\right)$, comme sur le champ de bataille, grâce aux innovations : «L'artillerie de campagne de France, est aujourd'hui si mobile, les canonniers ont été si exercés pendant la paix, et ils ont d'ailleurs tant d'avantages avec leur bricoles, pour la manœuvrer lestement, qu'il n'y a pour ainsi dire, point de terrain qui puisse retarder les opérations ${ }^{40}$. » Ainsi, les deux traducteurs-artilleurs représentent les deux parties du débat, puisque le marquis de Saint-Auban fut, au contraire, l'un des principaux opposants aux réformes de Gribeauval. Les autres traductions sont de qualité inférieure et les traducteurs bien plus effacés : la main du traducteur de Flavigny ${ }^{41}$ se trouve surtout dans le titre des Principes fondamentaux de la construction des places, avec l'insistance sur la nouveauté du système de fortification et de la théorie des mines qui a clairement un but promotionnel. Mais, en général, une certaine négligence peut être relevée: l'Examen de la poudre de Flavigny contient non seulement quelques imprécisions, qui compromettent la compréhension du texte, mais également beaucoup d'erreurs dans les formules mathématiques ${ }^{42}$. Bien qu'en moindre quantité, de telles erreurs sont également présentes dans la traduction de De l'Usage des armes à feu réalisée par Saint-Auban ${ }^{43}$. Toutes ces traductions comportent, enfin, des simplifications syntaxiques pour passer de la complexité de la langue italienne à la « langue de la clarté ».

21 Toutefois, quelques altérations volontaires du texte par le traducteur peuvent être opérées. Ainsi, une intervention significative de Flavigny dans l'Examen de la poudre a pour but de masquer l'absence de collaboration entre les corps d'artillerie français et piémontais. En traitant des expériences sur les charges de poudres nécessaires dans une pièce d'artillerie, d'Antoni affirme :

Questo scopo di stabilire quale sia la carica, con cui si ha il tiro più lungo, siccome ha mosso gli Artiglieri Piemontesi a fare molte sperienze, così ha rivolto la scuola dell'Artiglieria Francese a farne delle altre, delle quali brameremmo pure poterne fare il paragone con quelle, che si sono descritte finora, null'altro essendo delle medesime a notizia nostra pervenuto, se non il credersi, che ne' pezzi di gran calibro sia questa carica circa $3 / 8$ del peso della palla ${ }^{44}$.

On pourrait ainsi traduire au pied de la lettre ce passage, qui dénote la tension entre la libre communication des données scientifiques et les éventuelles restrictions de la circulation des informations militaires :

Beaucoup d'expériences ont été faites par les artilleurs piémontais dans le but d'établir quelle est la charge avec laquelle on tire plus loin. L'école d'artillerie française en a également fait d'autres, qu'on aimerait bien pouvoir comparer avec celles décrites jusque-là, mais la seule information qui nous soit parvenue, est que l'on croit que, dans les pièces de grand calibre, cette charge est d'environ $3 / 8 \mathrm{du}$ poids du boulet.

Or, Flavigny se contentait de traduire : «Les Artilleurs Piémontais ne sont pas les seuls qui aient fait des expériences pour découvrir les charges qui donnent les plus longues 
portées ; mais ce qui en est parvenu jusqu'à présent à ma connaissance ${ }^{45}$... » Il ne faisait donc ici aucune référence explicite aux expériences françaises ni commentaire qui pût éclairer le lecteur français sur ce point, soit qu'il gardât le secret sur leurs résultats, soit qu'il n'en eût lui-même pas connaissance, puisqu'il n'appartenait pas au Corps royal de l'Artillerie. Mais l'occultation ne concernait finalement que ces résultats et leur noncommunication puisque, dans la même page, on explicite qu'il s'agissait: "des expériences faites par les François ${ }^{46}$. ».

\section{Une unique traduction du français en italien}

Dans le seul ouvrage traduit en italien du français dans la seconde moitié du XVIII siècle, L'Artillerie raisonnée de Guillaume Le Blond, le traducteur Gabriele Zener, capitaine des ordinanze (milices) de la République de Venise, assurait que : «n'ayant pu souffrir, que ce travail, quel qu'il soit, ne se bornât qu'à une simple traduction... peu satisfait, du seul nom d'interprète, j'ai voulu enrichir l'œuvre de tables, de figures et de notes importantes, tirées des meilleurs sources ${ }^{47}$ ». Il s'agissait manifestement de sa part d'une stratégie pour élargir le cercle de ses lecteurs potentiels: par cet enrichissement personnel, le traducteur espérait pouvoir attirer tous les artilleurs, et non pas simplement ceux qui ne connaissaient pas assez la langue française pour lire l'original. Le traducteur s'engageait aussi, si son entreprise était couronnée de succès, à traduire deux autres ouvrages du même auteur - ce qui n'eut jamais lieu. Ceci pourrait s'expliquer non seulement par l'absence de public pour ce type d'ouvrages, mais également par la nature même de la traduction proposée, qui ne maintenait que partiellement ses promesses : elle se contente de calquer l'original, en maintenant fidèlement sa structure, sans rajout ni coupure ${ }^{48}$. Même si le traducteur a eu soin de rajouter de nombreuses notes (cent soixante au total), plus des trois quarts d'entre elles concernent les conversions de mesures ${ }^{49}$, indispensables au lecteur italien, alors que la majorité des traducteurs se contentaient des tables de conversions. Deux notes seulement expriment une critique. Dans la plus importante, Zener s'abstient de prendre parti dans un "conflit» entre trois autorités: Le Blond attribuait la méthode de placer plusieurs niveaux de fourneaux de mine dans un siège, afin de multiplier l'effet des mines explosive, à Vallière, mais le chevalier de Folard en avait écrit « de façon, qu'il paraît, qu'il eut été le premier à le deviser. Moi, je transcris ici ses propres mots en français, pour que chacun puisse juger soi-même ${ }^{50}$. " Finalement, seule une vingtaine de notes comportent des additions ou des explications. Les additions étaient essentiellement extraites des œuvres déjà citées par l'auteur (Saint-Rémy, Bélidor, Folard, l'Encyclopédie), mais que ne connaissait pas nécessairement le lectorat italien, qui pouvait ne pas posséder ces œuvres, si populaires fussent-elles. Tout en demeurant neutre, Zener avait également ajouté des extraits par d'autres auteurs faisant autorité, tels que Vallière et Dulacq, procédant par accumulation érudite plutôt que par une approche critique.

\section{La présence française et le Risorgimento}

Entre la fin du XVIII ${ }^{e}$ et le début du XIX ${ }^{e}$ siècle, la présence française en Italie favorisa la circulation des hommes et des savoirs, y compris dans le domaine militaire. Le modèle français, notamment en ce qui concerne les armes savantes, se fit dominant pendant l'époque napoléonienne, Bonaparte lui-même étant issu de l'artillerie. Le processus 
d'acculturation militaire de l'Italie se fit également par le biais des traductions. Il n'est pas surprenant que celles-ci soient attestées dans des territoires qui demeurent formellement indépendants, comme la République italienne et le Royaume d'Italie. Ce modèle scientifique et technique - et souvent politique - fut durablement présent dans la première phase du Risorgimento, même après le départ des Français, notamment jusqu'aux années 1830 .

\section{La République Italienne et le Royaume d'Italie}

Pendant la République Italienne et les premiers mois du Royaume d'Italie, on observe une forte concentration de traductions, avec trois traductions d'ouvrages français en deux ans (1804-1805). Cet accroissement s'explique, comme on l'a vu, par la présence française, mais aussi par la nouvelle centralité acquise par le domaine militaire, avec la formation de la nouvelle armée italienne. Cette période et ce contexte, en effet, se révèlent cruciaux, car, dans l'organisation des corps savants italiens, beaucoup sont des officiers exilés, notamment napolitains, qui contribueront par la suite au rayonnement du modèle français dans la péninsule ${ }^{51}$.

Le premier texte, strictement technique, reste anonyme - tant pour l'auteur que pour le traducteur. En 1804, la traduction du Petit manuel du canonnier sortit également à Milan. On peut supposer que cette entreprise répondait à la nécessité d'un manuel portatif, puisqu'il ne comptait que cent vingt pages, soit le dixième des mille deux cents pages de l'œuvre la plus largement consultée pour le service, l'Aide-mémoire à l'usage des officiers d'artillerie de France attachés au service de terre du général Gassendi. Celui-ci ne fut jamais traduit, mais nous n'avons pas d'informations sur l'usage du Petit manuel du canonnier, qui n'a pas été commandé pour la bibliothèque de la nouvelle école régimentaire d'artillerie, pourtant créée à Pavie la même année ${ }^{52}$. Une traduction du même texte par l'artilleur Pietro De Angelis est signalée : elle sortit en 1810 à Naples, mais l'on a perdu les traces de cette œuvre ${ }^{53}$.

Une autre traduction de cinquante-trois pages seulement, sortie en 1805 , fut celle du texte écrit par le général Dembarrère du Coup d'œil pour distinguer et classer les différentes parties de la science militaire ou de l'Art de la guerre sur terre, sorte de panégyrique des ingénieurs militaires. Ne pouvant plus servir activement, mais visant une place dans le génie, le capitaine Giulio Pampani ${ }^{54}$ (1757-1810) en dédia sa traduction au ministre de la guerre; il atteignit partiellement son objectif l'année suivante en entrant dans les sapeurs. Enfin, l'autre traduction retrouvée pour la période napoléonienne est celle d'un ouvrage de peu d'intérêt, les Memorie d'Artiglieria, de soixante pages, publié, en 1805 également, par le lieutenant en second de l'artillerie italienne Antonio Corso: il s'agit d'un recueil de mémoires sur l'artillerie de "plusieurs auteurs français » ${ }^{55}$, mais dont la moitié est en fait extraite de l'ouvrage de Le Blond précédemment cité - même si rien n'indique que Corso ait eu accès à la traduction de Zener - et d'un autre texte du même auteur ${ }^{56}$. Là encore, à l'exception de courtes préfaces, les deux traducteurs, Pampani et Corso, s'abstiennent de toute intervention dans le texte traduit.

Nous savons en outre que, en 1812, un ouvrage plus consistant avait obtenu du ministère de la guerre l'autorisation d'impression. Apparemment, il aurait été imprimé en deux cent quatre-vingt exemplaires au moins, mais les traces de ses deux volumes ont été perdues : il s'agit des Élémens de topographie militaire de l'ingénieur prussien Hayne ${ }^{57}$. Or, la traduction réalisée par le lieutenant d'artillerie Luigi Fortis était fondée sur le texte 
français du capitaine du génie Bayart, et non sur l'édition originale en allemand. On a donc ici un premier cas de retraduction.

\section{Les traductions pour l'artillerie du royaume des Deux-Siciles}

30 Si l'agrégat des trois traductions qui précèdent apparaît plus significatif pour leur datation que pour le contenu, en revanche les trois traductions publiées dans le royaume des Deux-Siciles sont importantes également à cause de leur contenu scientifique et technique. Les deux premières sont publiées en 1816, juste après que les Français aient été chassés du Royaume. Elles témoignent cependant de l'intérêt persistant pour leurs méthodes, qui avaient notablement enrichi l'artillerie napolitaine ${ }^{58}$. Le Traité $d u$ mouvement des projectiles, appliqué au tir de bouches à feu et les Tables du tir des canons et des obusiers de Jean-Louis Lombard présentaient respectivement le cadre théorique et les outils fondamentaux de la balistique extérieure, qui avaient été utilisées pour le pointage des canons tout au long des guerres de la Révolution et de l'Empire ${ }^{59}$ et constituaient en quelque sorte un couronnement de la «révolution balistique». Leur traduction fut entreprise par le lieutenant-colonel d'artillerie napolitain Giovanni Battista Pacces ${ }^{60}$, professeur à l'école d'application d'artillerie de Capoue. Le fait que, jusque-là, ces textes avaient été utilisés en français confère sans doute à ces traductions un caractère d'adaptation «nationale ».

31 On reste dans le même cadre pour le Traité théorique et pratique des Batteries du capitaine d'artillerie Lamy, traduit en italien par son homologue napolitain Novi en 1830. Ce texte répondait au besoin spécifique d'un traité sur la construction des batteries, en France comme dans le royaume des Deux-Siciles, où le tirage du traité publié par Pacces en 1813 était épuisé ${ }^{61}$. Le traducteur souligne la valeur du texte de Lamy et la nécessité de le faire traduire pour en assurer la diffusion dans le royaume, entreprise à laquelle il avait été encouragé par ses collègues et supérieurs ${ }^{62}$. Ce patronage officiel est d'ailleurs évident d'après l'éditeur même de cette traduction : la Reale Tipografia della Guerra. Le traité de Lamy est sans doute le texte dans lequel la main d'un traducteur italien est la plus visible. Tout d'abord il fit, comme Zener, les conversions des mesures, en les insérant cependant dans le texte et en supprimant ainsi les mesures originales - ce qui constitue un cas unique dans notre corpus. Ce choix, qui rétrécissait le nombre de lecteurs potentiels, puisqu'ils devaient avoir connaissance des mesures napolitaines, renforce cependant le caractère national de la traduction et son usage pratique. Ensuite, son intervention dans ses notes de commentaires insérées en fin d'ouvrage est significative. Il s'agit de dix-neuf notes couvrant au total vingt-trois pages et à peu près équitablement réparties entre des notes où le traducteur exprime son opinion, des additions et des explications ${ }^{63}$. Les notes d'explication pouvaient détailler des calculs ou des démonstrations géométriques, ce qui, une fois encore, ne pouvait pas manquer de poser des problèmes de compréhension à un lectorat moins instruit que celui existant en France. Il y a enfin des prises de position du traducteur par rapport à certaines questions techniques qui, parfois, contrastent avec celles de l'auteur. Par exemple, Novi contestait la méthode proposée par Lamy pour calculer la distance de tir d'une pièce, basée sur la différence entre la vitesse de la lumière et celle du son: non seulement Lamy ne tenait pas compte des dernières expériences faites par le Bureau des Longitudes sur la vitesse du son, mais il suggérait de compter ses propres battements cardiaques pour déterminer une minute, en assumant arbitrairement 
soixante pulsations par minute. Cependant, Novi ne trouvait rien de mieux que de suggérer l'usage d'une montre à seconde ${ }^{64}$.

\section{La réactivité de la Toscane} partir de sa traduction française par deux artilleurs : le même Ravichio de Peretsdorf et le capitaine Nancy. Il s'agissait là d'une œuvre collective conçue au sein du corps de l'artillerie française, puisque les traducteurs avaient collaboré avec le général de division Gassendi, auteur de l'Aide-mémoire déjà mentionné, ainsi qu'avec le colonel Marion, inspecteur des fonderies ${ }^{69}$. Le texte original fut ainsi réaménagé, avec des additions et des coupures pour l'adapter aux besoins de l'artillerie française. Cette polyphonie de l'édition française, qui tient aux notes écrites par ces quatre officiers, a été strictement conservée dans la traduction italienne, car Biondi Perelli ajouta seulement six notes pour les trois volumes $^{70}$. Son unique critique n'est d'ailleurs pas dénuée de visées nationales : alors que les auteurs présentent la France comme le berceau de l'art de la fortification, le traducteur observe: "Chacun sait que les Français ont appris des Italiens les fortifications et que Vauban s'attribua les inventions du bolonais De Marchi ${ }^{71}$. " Il est d'ailleurs intéressant de comparer les notes d'origine des quatre officiers français, car les critiques sont presque exclusivement exprimées par les officiers les plus gradés, le colonel Marion et le général Gassendi. Une fois encore, c'est donc la déférence qui prime, et l'on comprend mieux l'absence d'interventions du lieutenant italien, qui se limita à traduire très fidèlement. 

témoignent ses autres traductions: son intervention dans le Cours élémentaire de fortification par Savart se limitait tout au plus à quelques pages de définitions supplémentaires ou à l'abréviation d'un chapitre ${ }^{72}$. Notons que, hormis le Traité de pyrotechnie militaire, ces ouvrages étaient généralement moins destinés aux artilleurs qu'aux officiers des autres armes. Cela témoigne sans doute d'un niveau inférieur de l'instruction de l'artillerie toscane par rapport à l'artillerie française. Ceci est confirmé par la « Préface du traducteur » de l'Instruction sur la balistique, dans laquelle Biondi Perelli explique avoir choisi ce traité parce qu'il s'agit du seul qui suive les lois de Galilée « sans recourir au calcul intégral ou différentiel », et puisse résoudre les problèmes "par le moyen d'une seule équation » et sans tables de logarithmes ${ }^{73}$. De fait, un demi-siècle après les œuvres de d'Antoni, qui avaient été traduites de l'italien pour leur caractère novateur dans le cadre d'un changement de paradigme de la balistique entre les sciences galiléenne et newtonienne, comme on l'a dit plus haut, on traduisait encore du français des œuvres qui demeuraient dans le paradigme galiléen, mais qui avaient au moins l'avantage de la simplicité.

Il n'empêche que l'envergure des travaux de Biondi Perelli - qui au total traduisit quatorze volumes - fut appréciée. L'éminent écrivain militaire Mariano d'Ayala considère que »ce sont parmi les meilleures traductions vers l'italien d'ouvrages techniques militaires, et parmi les premières à contribuer à la restauration de la langue militaire italienne $^{74}$ ». Ainsi, par la traduction, ce personnage aujourd'hui totalement méconnu aurait contribué de façon notable à la culture militaire du Risorgimento.

\section{Les traductions des ingénieurs civils : Bélidor}

Si les traducteurs militaires d'ouvrages techniques se caractérisent, globalement, par leurs interventions, souvent limitées, dans le paratexte, qu'en est-il des civils confrontés à la même tâche ? C'est ce qu'on peut appréhender à travers les traductions de Bélidor : ses ouvrages, destinés à l'origine aux ingénieurs tant militaires que civils, sont en effet explicitement «convertis » pour les ingénieurs civils dans le Lombardo-Vénitien et en Toscane, ce qui est emblématique de l'évolution de ces professions - dans une dynamique qui est d'ailleurs bien connue ${ }^{75}$. En effet, les opportunités offertes par le libre exercice de la profession d'ingénieur civil, dans le contexte du renouvellement de la culture technique et scientifique et d'une réorganisation des structures universitaires, expliqueraient ces entreprises ${ }^{76}$ et, par conséquent, la «longévité » de Bélidor, plus importante en Italie qu'en France. Bélidor publia La Science des ingénieurs en 1729 et l' Architecture hydraulique entre 1737 et 1739. Ces ouvrages connurent une fortune remarquable et leurs dernières éditions en France eurent lieu, respectivement en 1830 et en 1819, par Claude-Louis-Marie-Henri Navier (1785-1836), académicien des sciences, professeur et, surtout, célèbre ingénieur des Ponts-et-Chaussées. Ces éditions conservaient «intact » le texte de Bélidor, mais en l'enrichissant d'un remarquable apparat de notes de critique et de mises à jour. Ce choix de Navier relevait du désir de préserver la valeur pratique $d u$ texte, tout en signalant les nombreuses erreurs théoriques qu'il contenait, eu égard aux importants progrès enregistrés en la matière depuis l'époque de sa publication ${ }^{77}$. Or, en Italie ces textes ne furent traduits qu'à partir de ces dernières éditions : en 1832 pour La Science des ingénieurs, et entre 1835 et 1839 pour l'Architecture hydraulique. Ces ouvrages faisaient partie de la collection Bibliothèque choisie 
de l'ingénieur civil, dirigée par l'ingénieur milanais Giuseppe Cadolini, qui comprenait aussi les traductions des textes de l'ingénieur des Ponts-et-Chaussées Sganzin et des architectes Quatremère de Quincy et Rondelet. Ceci prouve donc que l'école d'ingénieurs française est bel et bien restée un véritable modèle en Italie, bien au-delà de l'époque napoléonienne et du contexte militaire.

Or, il est certain que le destinataire de ces ouvrages est avant tout l'ingénieur civil : dans la traduction de La Science des ingénieurs par Luigi Masieri, le type de note le plus fréquent est l'explication des termes militaires élémentaires, parmi lesquels «batterie », "à l'épreuve de la bombe », « chemin couvert », « cortine » et " tenaille » ${ }^{78}$. La particularité des éditions italiennes, par rapport aux éditions françaises, réside dans le fait que l'apparat critique de Navier se trouve séparé du texte ${ }^{79}$. Ainsi, dans La Science des ingénieurs, trois textes sont juxtaposés : d'abord, celui de Bélidor qui apparaît largement périmé; ensuite, l'appendice, rajouté par le traducteur, qui contient les mémoires d'ingénieurs milanais ${ }^{80}$; enfin les notes de Navier. La deuxième édition, de 1840 , casse cette tripartition, l'appendice étant désormais mélangé aux notes de Navier, peut-être pour en renforcer l'autorité. C'est également le choix de Basilio Soresina, traducteur de l' Architecture hydraulique : ses notes et celles de Cadolini sont également séparées du texte et mélangées à celles de Navier ${ }^{81}$. Mais cette nouvelle entreprise éditoriale rencontra en 1836 des problèmes en raison de la mort de Navier, celui-ci n'ayant pu commenter les tomes successifs de cette œuvre. Dans le tome II de 1837, le traducteur s'engageait vis-àvis des souscripteurs à rédiger lui-même un apparat critique, en priant ses lecteurs de lui " épargner les conséquences d'une comparaison ${ }^{82}$ ». En réalité, il s'y soustrait lui-même, puisque les tomes II, III et IV ne contiennent que la traduction du texte de Bélidor, obsolète et sans aucun paratexte. Ce qui frappe le plus dans ces éditions italiennes, c'est l'acceptation, quoique masquée, de l'erreur. Dans l'« Avertissement» du tome II, il est admis naïvement que les erreurs de Bélidor, «nous l'espérons, ne sont pas dans notre traduction $^{83}$ » - ce qui revient à souligner l'absence de contrôle. Cette " confession » peut aisément être comparée à la déclaration d'intentions formulée dans la «Note des Éditeurs » de La Science des ingénieurs de 1832 :

On trouvera également signalées ici et là les erreurs très fréquentes et de tout genre de l'édition française de 1830 (dirigée par un certain Navier et publiée par un certain Didot), qui a religieusement copié les fréquentes erreurs commises dans l'édition de Charles-Antoine Jombert, pour obtenir l'indulgence de tous ceux qui, inexorables dans leur jugement des éditions italiennes, sont prêts à prendre pour argent comptant les éditions étrangères ${ }^{84}$.

Or, s'il y a des corrections par rapport à l'édition française - ce qui ne semble pas être le cas -, elles ne sont sûrement pas signalées. De même, dans la deuxième édition de 1840, il n'y a aucune trace de plusieurs corrections d'erreurs annoncées dans la » Préface » par rapport à la première édition ${ }^{85}$. Les deux éditions ne diffèrent que par quelques notes marginales ${ }^{86}$ et par l'addition de nombreux articles dans l'appendice. Cela relève probablement plus de la logique commerciale et d'une certaine paresse des traducteurs que du respect de l'auteur. C'est cependant un aspect important, même dans un contexte non-militaire: pour ces ingénieurs confrontés au problème de la réélaboration d'une culture dominante, il semble que la connaissance procède par accumulation plutôt que par critique.

41 Telle serait une conclusion que l'on pourrait tirer de l'ensemble du corpus. En règle générale, la préservation du texte original prime sur l'opération de transfert linguistique pour des nécessités différentes ou nouvelles. Il ne semblerait donc pas y avoir de 
spécificité de la traduction technique militaire, ni a priori de la traduction technique. L'analyse concorde en effet avec les conclusions des historiens qui ont travaillé sur les traductions en général ou sur la censure : dans la période considérée, le commentaire, si présent, se limite au paratexte. Cela relève de plusieurs facteurs : tout d'abord, du respect affiché envers l'autorité militaire et scientifique; ensuite, de l'habitus d'écriture administrative dans l'exposé formaté des activités quotidiennes et de la correspondance de bureau, où souvent l'intervention n'est possible que par addition en marge; enfin, de certaines contraintes éditoriales, qui imposent parfois des espaces ou des délais réduits. Les traducteurs militaires ne s'efforcent pas d'adapter le savoir, même quand leurs écrits sont destinés aux élèves ingénieurs ou artilleurs ${ }^{87}$. À partir de ce corpus, il ne semble pas $\mathrm{y}$ avoir de corrélation particulière entre le grade du traducteur et son degré d'intervention dans le paratexte, ni entre ce dernier et la période de la traduction ${ }^{88}$. De leur côté, les traducteurs civils opèrent d'une façon similaire. Civils ou militaires, la plupart des traducteurs de ce corpus sont des techniciens qui abdiquent leur rôle d'intermédiaire, en se contentant de l'invisibilité.

En ce qui concerne la distribution des traductions, elle reflète l'usage et la compréhension des langues française et italienne, la diffusion et la réception d'un modèle, piémontais d'abord, et surtout d'un modèle français. Bien qu'il ne soit plus politiquement neutre depuis la Révolution française, ce dernier connaît pourtant un rayonnement durable dans le domaine des transferts culturels à partir du début du XIX ${ }^{e}$ siècle. Ses liens avec le Risorgimento sont profonds mais complexes, a fortiori dans le cas de techniques militaires. Il est donc à regretter d'autant plus que les informations sur l'usage de ces traductions soient si limitées.

En tout cas, ces traductions participent du processus de professionnalisation des ingénieurs dans l'espace italien, qu'ils soient militaires ou civils. Le processus ne commence pas avec la Révolution - comme le démontre le cas du Piémont - mais il connaît un essor avec sa pénétration dans l'espace italien. La République italienne puis le Royaume d'Italie notamment constituent à la fois un pôle d'attraction et un creuset pour les ingénieurs militaires de la péninsule ${ }^{89}$. Il n'est donc pas anodin que l'inversion de tendance dans les traductions commence à partir de cet espace, pour se poursuivre pendant le Risorgimento.

\section{NOTES}

1. Ken ALDER, Engineering the Revolution: Arms and Enlightenment in France, 1763-1815, Princeton (NJ), Princeton University Press, 1997.

2. Janis LANGINS, Conserving the Enlightenment, French Military Engineering from Vauban to the Revolution, Cambridge, Massachusetts et Londres, M.I.T. Press, 2003. En France, ce système a fortement inspiré l'ingénieur Julienne de Belair, dont les Élémens de fortification ont été traduits et abrégés par Jonathan Williams, chef des ingénieurs américains et premier directeur de West Point. Cf. Patrice BRET, L'État, l'armée, la science. L'invention de la recherche publique en France, 1763-1830, Rennes, PUR, 2002, p. 381. 
3. Voir par exemple Michèle VIROL, «La traduction des ouvrages des ingénieurs : stratégies d'auteurs, pratiques des libraires et volonté des princes (1600-1750) », dans Artefact. Techniques, histoire et sciences humaines. L'Europe technicienne XV'-XVIII ${ }^{e}$, Rennes, PUR, n 4, 2016, p. 181-195, ou, dans le présent volume, les articles de Ferenc тотн pour la Hongrie ou de Kostas CHATzis pour la Grèce.

4. Les indications les plus précieuses pour cette période se trouvent dans des études d'histoire de l'édition: Luigi Mascilli MIGLIORINI, Gianfranco TORTORELli (dir.), L'editoria italiana nel decennio francese. Conservazione e rinnovamento, Milano, FrancoAngeli, 2016 ; ainsi que dans des travaux sur la censure : Domenico Maria BRUNI, "Con regolata indifferenza, con attenzione costante" Potere politico e parola stampata nel Granducato di Toscana (1814-1847), Milano, FrancoAngeli, 2015). Voir aussi Lorenzo CUCCOLI, « Le armi dotte tra Francia e Italia 1796-1814 », thèse de l'Università di Bologna et de l'Université de Paris I Panthéon-Sorbonne, 2012.

5. Giovanni Dotoli, Vito CASTIGLIONE MINISCHETTI (dir.), Les traductions de l'italien en français au XVIII siècle, Fasano, Schena - Paris, Presses de l'Université de Paris-Sorbonne, 2003 ; id., Les traductions de l'italien en français au XIX ${ }^{e}$ siècle, Fasano, Schena - Paris, Presses de l'Université de ParisSorbonne, 2004.

6. http://opac.sbn.it/opacsbn/opac/iccu/avanzata.jsp

7. Qui méritent une étude à part: par exemple, Corso di mattematiche ad uso delle scuole militari compilato dai professori di mattematiche Allaize, Billy, Puissant, Boudrot, Livorno, dalla tipografia e litografia di Giulio Sardi, 1830-1831, 4 vol., traduit par Ferdinando Biondi Perelli ou encore les manuels classiques de Bossut et Bézout ou celui, plus ancien, de l'abbé Deidier, Elementi generali delle principali parti delle matematiche, necessarj ancora all'artiglieria, e all'arte militare, Venezia, Modesto Fenzo, 1761-1762, traduit par Arduino et Matteo Dandolo. Les traducteurs de ces œuvres n'étaient d'ailleurs à l'évidence pas des militaires.

8. Dell'arme odiernamente usate in guerra; Nomenclatura delle altre parti del cannone; Tavola delle dimensioni, e peso dei cannoni; Diametro esteriore delle canne da fucile, moschettone, carabina, pistolla, $e$ del loro peso, e prezzo ; Annotazioni concernenti il fucile; Delle materie che compongono la polvere da guerra, e da caccia ; Della fabbricazione, e prova della polvere ; Esercizio in dettaglio del pezzo di cannone da 4 per l'istruzione dei cannonieri principianti.

9. Voir les recherches en cours de Maria Pia Casalena. Nous écartons dans le tableau quelques autres traductions attestées, que nous signalerons dans le texte, mais dont aucun exemplaire n'a pu encore être repéré dans les bibliothèques italiennes.

10. Alessandro Vittorio PAPACINO D'ANTONI, Esame della polvere, Torino, Stamperia reale, 1765 ; Alessandro Vittorio PAPACINO D'ANTONI, Dell'uso delle armi da fuoco per le regie scuole teoriche d'artiglieria, e fortificazione, Torino, Stamperia Reale, 1780 ; Jean-Louis LOMBARD, Tables du tir des canons et des obusiers, avec une instruction sur la manière de s'en servir, s.l., 1787 ; Jean-Louis LOMBARD, Traité du mouvement des projectiles, appliqué au tir de bouches à feu, Dijon, Imprimerie de L. N. Frantin, 1796 ; [Benjamin POUMET], Instruction sur la balistique, à l'usage des élèves du Corps royal d'État-major, Paris, Anselin et Pochard, 1824

11. Alessandro Vittorio PAPACINO D'ANTONI, Dell'artiglieria pratica per le Regie Scuole d'artiglieria, e fortificazione, t. II, Incumbenze degli artiglieri in tempo di guerra, Torino, Stamperia Reale, 1775 ; Guillaume LE BLOND, L'Artillerie raisonnée, contenant la description \& l'usage des différentes bouches a feu, avec les principaux moyens qu'on a employés pour les perfectionner, la théorie \& la pratique des mines, $\& d u$ jet des bombes, et l'essentiel de tout ce que l'artillerie a de plus intéressant depuis l'invention de la poudre a canon, Paris, C.-A. Jombert, 1771 ; [anonyme], Petit manuel du canonnier, ou instruction générale sur le service de toutes les bouches à feu, en usage dans l'artillerie, Paris, Magimel, An II (1793-1794) ; Antonio corso (éd.), Memorie d'artiglieria, Pavia, Giovanni Capelli, 1805 ; Jean-Nicolas LAMY, Traité théorique et pratique des Batteries, Paris, Anselin et Pochard, 1827 ; Joseph RAVICHIO DE 
PERETSDORF (éd.), Traité de pyrotechnie militaire, comprenant tous les artifices de guerre en usage en Autriche, Paris, F.-G. Levrault, 1824.

12. Alessandro Vittorio PAPACINO D'ANTONI, Instituzioni fisico-meccaniche per le regie scuole d'artiglieria e fortificazione, Torino, Stamperia reale, 2 vol., 1773-1774.

13. Alessandro Vittorio PAPACINO D'ANTONI, Dell'architettura militare per le regie scuole teoriche d'artiglieria, e fortificazione. Libro terzo in cui si contengono le regole della fortificazione difensiva, e delle mine per le piazze di guerra, Torino, Stamperia reale, 1759; Jean DEMBARRERE, Coup d'œil pour distinguer et classer les différentes parties de la science militaire ou de l'Art de la guerre sur terre, surtout pour reconnoître l'étendue et l'influence de celles qui appartiennent à l'arme du génie, Paris, Magimel, 1801 ; Nicolas-Pierre-Antoine SAVART, Cours élémentaire de fortification, à l'usage de MM. les élèves de l'École spéciale militaire, Paris, Anselin et Pochard, 1825 [éd. orig. 1812].

14. Qui était l'éditeur d'un manuscrit autrichien, révisé et complété par des annotations du capitaine d'artillerie Vergnaud.

15. Antoine-Marie Augoyat (1783-1864), l'autre auteur présumé du texte, était capitaine du génie. 16. Pour LE BLOND : L'Artillerie raisonnée..., op. cit. et La Science des ingénieurs dans la conduite des travaux de fortification et d'architecture civile, Paris, F. Didot frères, 1830.

17. Flavigny allait traduire aussi, de l'espagnol, William Bowles, Introduction à l'histoire naturelle et à la géographie physique de l'Espagne, Paris, L. Cellot et Jombert fils jeune, 1776, et Correspondance de Fernand Cortès avec... Charles-Quint, sur la conquête du Mexique, En Suisse, Libraires associés, 1779.

18. Il s'agit probablement de Jean-Baptiste-Gaspard Cusset, seigneur de Montrozard.

19. Giovanni Battista PACCES, Trattato ragionato sulle diverse batterie, Napoli, tipografia del Consiglio di Stato, 1813.

20. Giulio PAMPANI, Breve metodo per stimare i terreni, e fabbriche con in fine il modo pratico per difendersi dalle inondazioni de' fiumi, ed altre cose necessarie ad istruzione di quelli, che s'incamminano in tali professioni, Ferrara, Giovanni Antonio Coatti, 1780 ; PAMPANI, Risposta data da Giulio Pampani a varj suoi amici intorno l'interogazione fattagli, se v' abbia modo di riparare gl' argini de fiumi dalle corrosioni dell'acqua, danneggiando poco, o nulla le campagne adiacenti, [Ferrara, 1781].

21. Notamment le ministre de la guerre Pino pour Pampani et les commandants du corps d'artillerie Paolo Calori et Giuseppe Giannetti pour Corso et Biondi Perelli, respectivement.

22. Voir Giovanni Battista PACCES, "All'intelligente lettore ", dans Lombard, Tavole del tiro de' cannoni e degli obici, Napoli, Masi, 1816. p. 5.

23. Voir CUSSET DE MONTROZARD, "Discours Préliminaire", dans d'Antoni, Institutions physicoméchaniques, Strasbourg, Bauer et Treuttel, 1777, vol. I, p. III.

24. Marco MERIGGI, « Prove di comunità. Sui congressi preunitari degli stati italiani ", in Storia d'Italia, Annali 26. Scienze e cultura dell'Italia unita, Torino, Giulio Einaudi, 2011 p. 7-36.

25. Michela MINESSO, L'ingegnere dall'età napoleonica al fascismo, in Storia d'Italia. Annali 10. I professionisti, Torino, Giulio Einaudi, 1996, pp. 261-267. Luigi BLANCo (dir.), Amministrazione, formazione e professione: gli ingegneri in Italia tra Sette e Ottocento, Bologna, Il Mulino, 2000.

26. Maria Iolanda PALAzzolo, «Geografia e dinamica degli insediamenti editoriali » et Mario INFELISE, « La nuova figura dell'editore », dans Gabriele Turi (dir.), Storia dell'editoria nell'Italia contemporanea, Firenze, Giunti, 1997, respectivement p. 11-54 et p. 55-76.

27. Sur les traductions pendant le Premier Empire voire les recherches en cours de Maria Pia Casalena. Sur la censure voire Domenico Maria BRUNI, "Con regolata indifferenza, con attenzione costante" Potere politico e parola stampata nel Granducato di Toscana (1814-1847), Milano, Franco Angeli, 2015.

28. Brett D. Steele souligne l'influence de d'Antoni : Brett D. STEELE, « Muskets and Pendulums : Benjamin Robins, Leonard Euler and the Revolution in Ballistics», Technology and Culture, 35, 1994, n 1-2, p. 348-382; STEELE, "Military "Progress" and Newtonian Science in the Age of 
Enlightenment ", dans Brett D. Steele, Tamera Dorland (dir.), The Heirs of Archimedes. Science and the Art of War through the Age of Enlightenment, Cambridge-London, MIT Press, 2005, p. 361-390.

29. Voir la « Notice historique sur les Services, les Ouvrages et la Vie privée de M. le Marquis de Saint-Auban, Commandeur de l'Ordre Royal de Saint-Louis, Lieutenant-Général des Armées du Roi », dans D’ANTONI, De l'Usage des armes à feu, op. cit., p. 3-36.

30. Jacques-Antoine Baratier, marquis DE SAINT-AUBAN, « Avertissement sur la traduction », dans Alessandro Vittorio PAPACINO D'ANTONI, De l'Usage des armes à feu, Paris, Lambert, 1785, p. 39.

31. FLAVIGNY, « Avant-Propos ", dans D'ANTONI, Examen de la poudre, Amsterdam, Marc-Michel Rey, 1773

32. MarCo BERETTA, Bibliotheca Lavoisieriana: The Catalogue of the Library of Antoine Laurent Lavoisier, Florence, Leo S. Olschki, 1995, p. 200.

33. Gaspard-Herman cotTY, "Notice historique sur le Corps Royal d'Artillerie ", dans Cotty, Encyclopédie méthodique. Dictionnaire de l'artillerie, Paris, chez Mme veuve Agasse, 1822, p. 272 et 275.

34. Voir CUSSET DE MONTROZARD, «Discours Préliminaire », op. cit., p. III. Et pourtant, dans le titre, le traducteur précise qu'il s'agit des écoles royales d'artillerie et du génie « de Turin », ce qui allait de soi dans l'original.

35. Même si, au début, il avait entrepris la traduction tout seul "pour [s']exercer dans une langue, dans laquelle on s'apercevra facilement [qu'il] débute». Voir CUSSET DE MONTROZARD, « Discours Préliminaire », op. cit., p. III-IV.

36. D’Antoni envisageait toutefois un public plus large que celui des élèves des écoles militaires de Turin. Il souhaitait ainsi que « ce qui appartenait à peu de gens se répande à tous, et que ce qui était presque privé, devienne commun et profite à chacun ». Voir D'ANTONI, Instituzioni fisicomeccaniche, op. cit., vol. I, p. v: « si diffonda su tutti, e ciò, ch'era di pochi proprio, e quasi privato, comune divenga, e di pubblica ragione, e profitto a chicchessia ».

37. D'ANTONI, Du Service de l'artillerie à la guerre, Paris, L. Cellot, 1780, p. 15.

38. D'ANTONI, Du Service de l'artillerie à la guerre, op. cit., p. 14, dans l'« Introduction et plan de l'ouvrage », puis dans les notes aux p. 21, 191, 306, 307, 313, 330, 369 et 371. Les 115 notes sont ainsi reparties : 28,70\% sont constituées de l'expression de l'opinion du traducteur ; $28,70 \%$ sont constituées de notes d'explications ; $20 \%$ sont des mises à jour ; 7,83\%, des conversions ; 6,96\%, des additions ; 6,09\%, des descriptions des usages français.

39. Ibid., p. 21.

40. Ibid., p. 306.

41. Flavigny, qui avait participé à la guerre de Sept Ans, fut en mission en Piémont entre 1772 et 1773. Voir le Dictionnaire de biographie française, t. 13, Paris, Letouzey et Ané, 1971-1975, colp. 1506-1507.

42. On peut notamment en trouver aux pages 142, 157, 191, 206, 211, 216, 222, 228, 234 et 236.

43. Le décès $d u$ traducteur quand il allait terminer le travail pourrait expliquer l'absence de l'index et de l'adresse au lecteur, qui ne furent pas traduits, et peut-être aussi de notes du traducteur. Cf. « Notice historique sur les Services, les Ouvrages et la Vie privée de M. le Marquis de Saint-Auban, Commandeur de l'Ordre Royal de Saint-Louis, Lieutenant-Général des Armées du Roi », dans D'ANTONI, De l'Usage des armes à feu, p. 35.

44. D'ANTONI, Esame della polvere, op. cit., p. 121.

45. D’ANTONI, Examen de la poudre, op. cit., p. 109-110.

46. Ibid., p. 110.

47. Gabriele ZENER, « Avis du traducteur », dans Guillaume LE BLOND, L' artiglieria per principj, e per raziocinio, Trattato che contien la descrizione, e l'uso delle diverse bocche da fuoco, e i principali mezzi, che si sono adoperati per perfezionarle, La Teoria, e la pratica delle Mine, e del Getto delle Bombe, e tutto ciò, che l'Artiglieria ha di più importante dopo il trovato della polvere da Cannone, Venezia, Antonio 
Locatelli, 1773, vol. I,., p. x-xi : « non ho io potuto sofferire, che questa mia fatica, qualunque siasi, si ristringesse ad una nuda, e semplice Traduzione... poco contento del solo nome d'Interprete, ho io voluto arricchir l'Opera di Tavole, di Figure, e di Note importanti, cavate da' fonti migliori ".

48. L'index analytique, cependant, n'est pas présent dans la version italienne.

49. 123 notes $(76,88 \%)$ concernent les conversions de mesures ; 18 (11,25\%) sont des additions ; $17(10,63 \%)$ des explications et $2(1,25 \%)$ des critiques. Dans le deuxième volume (car la traduction en italien avait été réalisée en deux volumes), on rajoutait encore la traduction des deux textes, l'un sur l'histoire du corps d'artillerie en France et l'autre sur ses écoles régimentaires.

50. Le Blond, L' artiglieria per principj, e per raziocinio, op. cit., vol. II, p. 124 ; " in modo, che parrebbe, che fosse stato egli il primo ad immaginarlo. Io qui trascrivo le sue stesse parole in Francese, perché possa ognuno veder meglio da sé la cosa » - Ibid., p. 75). Dans l'autre note de critique, le traducteur précise qu'il suivait la première édition de 1761 , la deuxième comprenant des coquilles (Ibid., p. 124).

51. Lorenzo cuccolI, « Le armi dotte tra Francia e Italia 1796-1814 », op. cit.

52. Archivio di Stato di Milano, fonds Ministero della guerra, carton 122 : Inventario Generale delle Bocche a fuoco, Modelli, Armi bianche e da fuoco, Giuochi d'Armi, Macchine, Strumenti, Cordaggi, Biblioteca, e Generi Diversi esistenti nella Scuola Teorico Pratica d'Artiglieria in Pavia all'Epoca del 15 Marzo Anno 1804, e $3^{\circ}$ della Repubblica Italiana.

53. Mariano D'AYALA, Bibliografia militare italiana antica e moderna, Torino, Stamperia Reale, p. 144.

54. Pampani avait été ingénieur civil au service vénitien, puis du Pape, avant d'entrer dans le Bureau topographique napolitain. Il entreprit sa carrière militaire comme ingénieur-géographe dans la république napolitaine en 1799 et fut exilé en France. Il servit dans l'armée de la République italienne comme ingénieur-géographe de 1802 à 1804.

55. Antonio CORSO, « Il traduttore a' suoi fratelli d'arme » ("Le traducteur à ses frères d'armes »), dans Corso (dir.), Memorie d'artiglieria, op. cit., p. 1.. Voir note .

56. Il s'agit de LE BLOND, Traité de l'Artillerie, ou des Armes et Machines en usage à la Guerre, Depuis l'invention de la Poudre, Paris, C.-A. Jombert, 1743.

57. Ou instruction détaillée sur la manière de lever à vue et de dessiner avec promptitude les cartes militaires, Paris, Magimel, 1804. Voir dans l'Archivio di Stato di Milano, fond Ministero della guerra, carton 2849, la lettre du lieutenant en second Luigi Fortis au Ministre de la guerre, Mantoue, 23 mars 1812, ainsi que la note de Beroaldi, chef de la $3^{\mathrm{e}}$ division du ministère de la guerre, Milano, 18 juillet 1812.

58. Notamment depuis la mission à Naples du général Pommereul entre 1787 et 1795, qui y avait introduit de nouvelles techniques de fonte, ainsi que le système Gribeauval.

59. Sur les contributions de Lombard et ses tables, voir Brett D. STEELE, « Military "Progress" and Newtonian Science in the Age of Enlightenment ", art. cit., p. 376-378, et Brett D. STEELE, « Muskets and Pendulums: Benjamin Robins, Leonard Euler and the Revolution in Ballistics ", art. cit., p. 376-377.

60. Pacces était chargé de l'instruction des lieutenants élèves d'artillerie depuis 1810 dans le Royaume de Naples de Murat. Le traducteur analyse, dans sa préface (Giovanni Battista Pacces, « All'intelligente lettore ", dans LOMBARD, Tavole del tiro de' cannoni e degli obici, op. cit., p. 5-13), les avantages et les inconvénients de la méthode de Lombard et de ses tables de tir, en soulignant la nécessité de faire converger la théorie et l'expérience. C'était à peu près aussi l'avis du même Lombard dans sa «Préface » du Traité du mouvement des projectiles. Pacces ne rajoute pas de notes au traité, son intervention étant limitée à l'insertion des errata corrige français dans le texte. Cependant, il rajoute quatre notes aux Tables du tir des canons et des obusiers : deux de mises à jour, une d'explication et une d'addition. 
61. En ce qui concerne la France, Lamy affirme que le seul traité utile sur la question est l'Aidemémoire à l'usage des officiers d'artillerie de France attachés au service de terre du général Gassendi. Voir Jean-Nicolas LAMY, « Prefazione » (Préface) dans Trattato teorico e pratico delle batterie, Napoli, Reale Tipografia della Guerra, 1830, p. V. Il « oublie» l'œuvre de ses collègues Ravichio von Peretsdorf et Nancy, publiée seulement un an auparavant et qui, cependant, n'était pas adaptée au nouveau système Vallée, qui avait remplacé le système Gribeauval en 1827 : voir Maurice-Joseph-Didier RAVICHIO DE PERETSDORF, Anne-Philibert-François-Claude NANCY, Traité théorique et pratique de la construction des batteries, Paris, F.-G. Levrault, 1826. En ce qui concerne le royaume des Deux Siciles, Novi fait réference au Trattato ragionato sulle diverse batterie de Pacces: Pietro NOVI, « Il Traduttore a chi legge » (Le traducteur au lecteur), dans LAMY, op. cit., p. III. Pour l'ouvrage de Pacces, cf. note .

62. Ibid., p. III. " Pubblicatosi intanto in Francia il trattato delle batterie del Signor J. N. Lamy Capitano del Corpo Reale di Artiglieria di quel Regno, gli elogi che distinti Uffiziali dell'Arma qui ne faceano, ci indussero a credere che utile sarebbe stato il recarlo nell'italiano idioma, onde renderne agevole lo acquisto. E fummo in ciò incoraggiati e da' compagni, e da' superiori puranco, i quali utile e lodevole intrapresa la reputavano. » («Ayant été publié en France le traité des batteries de Monsieur J. N. Lamy, capitaine du Corps Royal d'Artillerie de ce Royaume, les éloges qu'en firent ici différents officiers de cette arme nous amenèrent à penser qu'il aurait été utile de le traduire en italien, afin d'en favoriser l'achat. On a été encouragé dans cette entreprise par des camarades et même par des supérieurs, qui ont réputé l'entreprise utile et louable. »).

63. Les notes de Novi sont constituées par 6 additions (31,58\%), 5 explications (26,32\%) et 6 notes $(31,58 \%)$ où le traducteur exprime son opinion. Dans deux d'entre elles, il se limite à constater qu'il n'a pas suffisamment d'informations sur le nouveau système d'artillerie française, de sorte qu'il ne peut pas donner son évaluation d'un passage du texte. Ainsi, pour la première, écrit-il : « In Francia si sono fatti importanti cambiamenti alle macchine di artiglieria, ma da noi non se ne conoscono ancora le particolarità. » ( En France d'importants changements ont été faits dans les machines de l'artillerie, mais chez nous, on n'en connait pas encore les détails. »; NOVI, « Note del traduttore ", dans LAMY, op. cit., p. 194). Il faut remarquer qu'il s'agit du seul livre traduit que nous ayons repéré sur l'artillerie datant de 1827 ou après et, donc, qui prenne en compte le nouveau système Vallée, ceux traduits par Biondi Perelli ayant comme date-limite 1825. Les notes de Novi ne concernent que la première partie de l'ouvrage, celle sur les batteries «permanentes », qui occupent du reste la plus grande partie de l'ouvrage, celle sur les batteries mobiles ne constituant qu'une vingtaine de pages. Novi rajouta cependant à cette deuxième partie des instructions de Fréderic II de Prusse à son artillerie. Voir « Istruzione per la mia Artiglieria sulla maniera di dirigere i suoi fuochi nelle occasioni : data a' 10 Maggio 1782 », dans « Note del traduttore ", Ibid., p. 211-215. Ces instructions, quoique datées, avaient une valeur pratique, et étaient en fait à peu près énoncées par Lamy dans ses «Precetti " (Préceptes) le troisième à la page 178 , le quatrième à la page 179 , les huitième, neuvième et dixième à la page 181.

64. Novi, « Note del traduttore ", op. cit., p. 201.

65. Auquel sont dédiés tous ces ouvrages. Biondi Perelli traduit également en 1831 les Cours de mathématiques (voir note ). Ces cours étaient a priori destinés aux officiers de la ligne, mais il est probable que, comme les quatre autres textes traduits, ils aient été également destinés aux élèves d'artillerie toscane.

66. Sur le français comme langue intermédiaire, voir Giovanni DотоLI, "Bibliothèque des traductions de l'italien en français $d u x I^{e}$ au xx ${ }^{\mathrm{e}}$ siècle. Un parcours de l'Europe de la culture.», dans Les traductions de l'italien en français $d u X^{e} I^{e}$ au $X x^{e}$ siècle, actes du colloque international (Monopoli, 4-5 octobre 2003), Fasano, Schena, 2004, p. 7-35, et Pascal CASANOVA, La République mondiale des Lettres, Paris, Seuil, 2008. 
67. Ravichio de Peretsdorf avait été au service piémontais et autrichien avant de passer au service français, où il était archiviste des bureaux de l'artillerie et du génie au ministère de la guerre.

68. Ibid., p. 106-109. Il s'agissait d'un passage de Robert Simmons, The Sea-gunner's Vade-mecum, London, Steel \& co., 1812, p. 206-209. Biondi Perelli éliminait également la Préface du traducteur et l'Avis pour rajouter des Prénotions historiques.

69. « Avertissement », dans VON DECKER, Traité élémentaire d'artillerie, op. cit., vol. I, p. i-ii.

70. Il ajouta une note d'explication, deux notes de conversion des mesures, une note de critique et deux notes bibliographiques (l'une de ses traductions et l'édition des Euvres du général Montecuccoli par Ugo Foscolo).

71. VON DECKER, Trattato elementare d'artiglieria per l'uso dei militari di tutte le armi, Livorno, Giulio Sardi, 1830, vol. II, p. 178 : " E' noto ad ognuno che i Francesi hanno dagl'Italiani appreso le fortificazioni e che Vauban a se stesso attribuì le invenzioni di De Marchi bolognese ».

72. Biondi Perelli rajoute des Definizioni (Définitions) (p. 1-29), essentiellement tirées du Dizionario Militare (Dictionnaire militaire) de Giuseppe Grassi et quatre pages d'Appendice alle definizioni (Appendice aux définitions), vraisemblablement écrites par lui. Il signale également qu'il a abrégé le chapitre IV, "Del modo di dirigere e incassare i fiumi " (De la façon de diriger et encaisser un fleuve) : voir Nicolas-Pierre-Antoine SAVART, Corso elementare di fortificazione ad uso delle scuole militari, Livorno, Giulio Sardi,,vol. I, 1830, p. 98. Dans cet ouvrage en quatre volumes, les notes de l'auteur et de l'éditeur français (le lieutenant-général du génie Pierre-AlexandreJoseph Allent) sont mêlées.

73. Ferdinando BIONDI PERELLI, « Prefazione del traduttore », dans Benjamin POUMET, Istruzione sulla balistica, Livorno, Pozzolini, 1827, p. 3-4. Biondi Perelli motive sa traduction par « l'absence de tout traité en langue toscane sur la balistique qui soit facile à comprendre pour les officiers de toute arme, et particulièrement de l'artillerie » («La mancanza in idioma toscano di un trattato sulla balistica tale che per la sua facile intelligenza riuscisse adattato agli uffiziali di ogni arma ed utile in special modo a quelli d'artiglieria »).

Dans cette même préface, Biondi Perelli louait les Cours de mathématiques à l'usage des écoles impériales militaires, qu'il traduisit par la suite, parce qu'il évitait presque d'utiliser le calcul différentiel et intégral. Dans ce texte, on trouve deux notes du traducteur: une addition ( $p$. 18-21) et une critique (p. 21-23).

74. Mariano D'AYALA, Bibliografia militare italiana antica e moderna, op. cit., p. 144. D'Ayala fait référence particulièrement aux deux retraductions.

75. Hélène VÉRIN, La gloire des ingénieurs : L'Intelligence technique $d u X_{V I}^{e}$ au XVIII ${ }^{e}$ siècle, Paris, Albin Michel, 1993 ; Patrice BRET, Irina GOUZEVITCH (dir.), La mise en place de la communauté internationale d'ingénieurs dans la première moitié du XIXe siècle, Paris, CRHST, 1997.

76. Maria Iolanda PALAzzolo, "Geografia e dinamica degli insediamenti editoriali », dans Gabriele Turi (dir.), Storia dell'editoria nell'Italia contemporanea, op. cit., p. 27. Sur les opportunités offertes pour la libre profession d'ingénieur dans le Lombardo-Vénitien, voir Bruno GIORDANO, Gli ufficiali della scuola militare di Modena (1798-1820): una ricerca prosopografica, Soveria Mannelli (CZ), Rubbettino, 2008, p. 144-146.

77. Charles C. GILLISPIE (dir.), Dictionary of Scientific Biography, New York, Charles Scribner's Sons, vol. I, 1981.

78. Dans l'édition de 1832 , on trouve 3 notes de mises à jour $(6,25 \%) ; 9$ d'additions $(18,75 \%)$ et 36 d'explications $(75 \%)$, dont $20(41,67 \%$ du total) pour des termes propres aux ingénieurs militaires. Elle fut imprimée d'abord à Milan, à Mantoue et à Florence (où le premier volume seulement fut imprimé, tandis que le deuxième le fut à Livourne en 1834). Une seconde édition sortit en 1840, avant qu'une troisième ne fut publiée à Milan et Naples en 1875-1876. 
79. À tel point que, dans les deux cas, sa traduction fut aussi publiée séparément : Claude-LouisMarie-Henri NAVIER, Note del signor Navier ingegnere di ponti e strade alla scienza degli ingegneri di Belidor, [s.l., s.d.] ; NAVIER, Note ed aggiunte del signor Navier ingegnere di ponti e strade alla architettura idraulica di Bernardo Belidor, Mantova, Negretti, 1843.

80. Notons qu'en marge du texte de Bélidor, on trouve toutefois quelques notes du traducteur, qui sont cependant souvent des explications ou des additions, ainsi que des renvois aux notes de Navier.

81. Bernard Forest DE BELIDOR, Architettura idraulica, Mantova, fratelli Negretti, vol. I, 1835.

82. DE BELIDOR, Architettura idraulica, Mantova, presso gli editori, 1837, vol. II, p. VIII, Avvertimento : « Risparmiare le conseguenze di un confronto.»

83. Ibid., p. vii : « errori che vogliamo sperare non sieno nella nostra traduzione.»

84. DE BELIDOR, La scienza degli ingegneri nella direzione delle opere di fortificazione e d'architettura civile, Mantova, Fratelli Negretti - Milano, Gaspare Truffi, 1832, vol. I, p. XI, « Nota degli Editori » : « Si troveranno pure accennati qua e là i moltissimi errori d'ogni fatta dell'edizione francese del 1830 (diretta da un Navier e pubblicata da un Didot) che ha religiosamente copiati i non pochi di quella di Carlo-Antonio Jombert del 1739 : valessero almen questi ad ottenerci indulgenza presso tutti coloro che inesorabili nel giudicare delle edizioni italiane sono prontissimi a trovare tutt'oro nelle straniere. "

85. DE BELIDOR, La scienza degli ingegneri, Milano, Perelli e Mariani, 1840, «Prefazione»: « On trouvera également corrigées plusieurs erreurs de la 1 ère édition italienne, erreurs inévitables dans des œuvres de longue haleine, dérivant en partie des nombreuses erreurs de tout type de l'édition française de 1830 " ( Si troveranno pure corretti varj errori corsi nella $1^{\circ}$ edizione italiana, errori inevitabili in opere di lunga lena, e che derivarono in parte dai moltissimi d'ogni fatta dell'edizione francese del $1830 »)$.

86. Ainsi, si une note a été supprimée, quatre autres ont été rajoutées, dont deux sont des additions au texte et deux contiennent les critiques suivantes : « La dite opinion n'est que celle de Bélidor " (" La qual opinione resti tutta a carico del Belidor »); « Bélidor fait référence à son époque » («Belidor si riferisce al tempo suo »).

87. Explicitement, dans le cas d'une des traductions de Montrozard, des quatre de Biondi Perelli et de deux de Pacces, soit sept sur seize. Bien évidemment, le nombre d'ouvrages effectivement présents dans les écoles est supérieur (voire par exemple note ).

88. Trois traducteurs interviennent plus significativement que les autres dans le paratexte: le lieutenant-colonel Montrozard dans Du Service de l'artillerie à la guerre, et les capitaines Zener et Novi. On ne retrouve donc certes pas les deux lieutenants, mais il en va de même avec le seul lieutenant-général. Les traductions concernées sont datées de 1773, 1780 et 1830.

89. cucCOLI, "Le armi dotte tra Francia e Italia 1796-1814 », op. cit. ; GIORDANO, Gli ufficiali della scuola militare di Modena, op. cit.

\section{RÉSUMÉS}

L'article examine les traductions de l'italien au français et du français en italien des ouvrages techniques destinés aux officiers des armes savantes entre 1750 et 1840 . Il en résulte une prévalence des traductions du français, mais avec une bipartition nette: au XVIII ${ }^{\mathrm{e}}$ siècle, on traduit plutôt de l'italien, tandis que, au XIX ${ }^{\mathrm{e}}$, on traduit plutôt du français. Les traducteurs, qui 
sont des officiers, souvent des armes savantes, peuvent écrire à l'usage d'écoles militaires et/ou viser une promotion. Ils restent cependant presque toujours très effacés, en se contentant d'intervenir dans le para-texte. Le respect du texte et de l'autorité militaire et scientifique de l'auteur demeure largement dominant, les critiques étant très rares, tandis que les additions dans le para-texte sont assez fréquentes.

The article examines the translations from Italian to French and from French to Italian of technical texts written for the use of military engineers and artillery officers from 1750 to 1840 . Translations from French to Italian dominate over the whole period, with a distinctive divergence between the $18^{\text {th }}$ century, with its prevalence of translations from Italian, and the $19^{\text {th }}$ , marked by the opposite trend. The translators are officers, often from the technical corps: they may write for military schools, but also in order to seek promotion. They remain largely invisible, confining their presence to the paratext. Respect for the text and for the military and scientific authority of the author is predominant, criticism being rare, while additions in the paratext are quite frequent.

\section{INDEX}

Mots-clés : traductions, génie, artillerie, français, italien

Keywords : translations, engineering, artillery, French, Italian

\section{AUTEUR}

\section{LORENZO CUCCOLI}

Membre associé de l'IHMC

Université de Bologne 